\title{
ANTIGENIC STIMULATION DURING PREGNANCY MODIFIES SPECIFIC IGA1 AND IGA2 SUBCLASSES IN HUMAN COLOSTRUM ACCORDING TO THE CHEMICAL COMPOSITION OF THE ANTIGEN
}

\author{
Erick S. Sánchez-Salguero ${ }^{1}$, Brenda C. Rodríguez-Chacón ${ }^{1,2}$, Jorge Leyva-Daniel ${ }^{1,3}$, \\ Josué Zambrano-Carrasco ${ }^{1,4}$, Carlos E. Miguel-Rodríguez ${ }^{1,5}$, and Leopoldo Santos-Argumedo ${ }^{1 *}$ \\ ${ }^{1}$ Department of Molecular Biomedicine, Center for Research and Advanced Studies, National Polytechnic Institute \\ (CINVESTAV-IPN), Mexico City, Mexico; ${ }^{2}$ Laboratory of Molecular Microbiology and Environmental Biotechnology, \\ Autonomous University of Guerrero (UAGRO), Chilpancingo, Gro., Mexico; ${ }^{3}$ Department of Biological Chemistry \\ Sciences, University of Sonora (UNISON), Hermosillo, Son., Mexico; ${ }^{4}$ Faculty of Biological Sciences, Autonomous \\ University of Puebla (BUAP), Puebla, Mexico; ${ }^{5}$ Department of Infectomics and Molecular Pathogenesis, \\ CINVESTAV-IPN, Mexico City, Mexico
}

\begin{abstract}
Background: Several studies have evaluated the effect of infectious diseases and vaccine protocols during pregnancy on maternal milk immunoglobulin ( $\mathrm{g}$ ) levels, to understand the protection conferred by lactation on newborns. Colostrum is the primary source of maternal IgA for the newborn. IgA participates in protection mechanisms in the neonate's mucosa. In humans, IgA has two subclasses with differential anatomical distribution among mucosal compartments. Total lgA levels in maternal milk vary after antigen stimulation and have differential affinities in function of the chemical composition of the antigens. We studied the effect of antigenic stimulation during pregnancy on the concentrations of specific $\operatorname{lgA} 1$ and $\operatorname{lgA} 2$ subclasses in human colostrum. Methods: We analyzed data from 113 women in Mexico City and compared the amount of IgA subclasses in colostrum against three antigens: two from vaccine protocols (tetanus toxoid and pneumococcal polysaccharides) and lipopolysaccharide, a ubiquitous antigen in the gastrointestinal tract. Results: In agreement with the previous reports, we showed that IgA1 from colostrum mainly recognized protein antigens; in sharp contrast, IgA2 was mostly directed against polysaccharide antigens. These levels increased in women who had previous contacts through vaccination or infections during pregnancy. Conclusions: Antigen interaction during pregnancy increased the amount of specific IgA subclasses, depending on the chemical composition of the antigen. (REV INVEST CLIN. 2020;72(2):80-7)
\end{abstract}

Key words: Immunoglobulin A1. Immunoglobulin A2. Colostrum. Tetanus toxin. Lipopolysaccharides. Pneumococcal polysaccharides.

*Corresponding author:

Leopoldo Santos-Argumedo

E-mail: lesantos@cinvestav.mx
Received for publication: 30-08-2019

Approved for publication: 17-10-2019

DOI: $10.24875 / R I C .19003230$

0034-8376 / (c) 2019 Revista de Investigación Clínica. Published by Permanyer. This is an open access article under the CC BY-NC-ND license (http://creativecommons.org/licenses/by-nc-nd/4.0/). 


\section{INTRODUCTION}

Tetanus toxin (TT) from Clostridium tetani is a protein that triggers muscular contraction, causing death. TT vaccine is formulated with an inactivated toxin (toxoid) that induces the production of neutralizing antibodies ${ }^{1}$. Pneumococcal polysaccharides (PPSs) are a complex of carbohydrates present in the capsule of Streptococcus pneumoniae bacteria. The capsule is an important virulence factor that inhibits phagocytosis, promoting infection in the respiratory tract. Pneumococcal vaccine is formulated with a pool of polysaccharides from different serotypes ${ }^{2}$. S. pneumoniae causes infrequent but severe infections in neonates ${ }^{3}$.

TT and PPS are two vaccines frequently administered during pregnancy to induce protection in women and newborns ${ }^{4}$. During intrauterine development, the fetus receives protection by transplacental transfer of immunoglobulin (Ig)G antibodies and, after birth, by maternal lactation containing mainly secretory IgA. Thus, antigen-specific lgs can be measured in serum and secretions such as colostrum and breast milk ${ }^{4}$.

Colostrum is the primary source of maternal $\lg A$. IgA has two subclasses in humans (IgA1 and $\lg A 2)^{5}$. In response to antigenic stimulation, IgA in the mucosa can be produced by T-dependent or T-independent mechanisms $^{6}$. Ladjeva et al. ${ }^{7}$ described significant individual variations of $\lg A 1$ and $\lg A 2$ subclasses in 18 colostrum samples. They analyzed IgA reactivity against polysaccharides and proteins, where $\lg A 2$ was more reactive against lipopolysaccharides (LPS); in contrast, the specificity for proteins was mainly found within $\lg A 1$.

Several controlled clinical studies have evaluated the effects of antigenic stimulation (vaccine protocols and infectious diseases) during pregnancy on colostrum and milk IgA levels ${ }^{8,9}$. In a previous study by our group, we found that infectious episodes in the gastrointestinal tract during pregnancy induce higher IgA2 levels in colostrum ${ }^{10}$. After recurrent gastrointestinal bacterial infections, LPS levels increase in the blood $^{11}$. LPS is a polysaccharide component of Gramnegative bacteria, and it is known to induce a strong systemic and local immune response by specific antibodies production ${ }^{12}$.
In this work, we analyzed the effect of antigenic stimulation on the levels of specific lgA subclasses in colostrum. Therefore, the main aim of our study was to evaluate the effects of vaccination or infection during pregnancy on the amount of specific colostrum IgA subclasses in a cohort of Mexican women. The quantitative enzyme-linked immunosorbent assay (ELISA) and Western blot techniques were used to measure colostrum IgA subclasses from 113 women in Mexico City, with an emphasis on IgA1 and IgA2 levels and their reactivity against specific antigens.

\section{METHODS}

\section{Calculation of population size (n)}

The population size $(n)$ was calculated considering the number of women who received anti-tetanus and anti-pneumococcal vaccines or had infectious episodes of the intestinal tract during pregnancy, 95\% confidence interval $(Z=1.96), 5 \%$ error.

\section{Population selection}

This study included colostrum samples from 113 pregnant, clinically healthy, women (Table 1 ) who delivered their babies at three large hospitals in Mexico City: Hospital Regional $1^{\circ}$ de Octubre ISSSTE (HR $1^{\circ}$ October), Hospital Regional de Alta Especialidad de Ixtapaluca 77 (HRAEI), or Hospital de la Mujer (HMuj). All procedures were explained to women, who signed assent and informed consent forms, following the Helsinki Declaration revised in $2013^{13}$. Clinical information related to the number of infectious episodes of the skin and respiratory, gastrointestinal, or urogenital tracts during pregnancy was obtained from each patient through an interview and clinical examination. Information depicted in the individual clinical files validated the data obtained from the interview at the time of sample collection.

\section{Inclusion and exclusion criteria}

Colostrum samples were obtained from women between 20 and 35 years of age, with a full-term pregnancy (38-42 weeks), with vaginal delivery (VD) or cesarean section (C-section), and, mainly, singleton births. Exclusion criteria were the presence of 
Table 1. Characteristics of the population studied

\begin{tabular}{ll}
\hline Characteristic & Frequencies (\%) \\
\hline Age of mothers & Mean $=27.4 \pm 4.58$ \\
Delivery method & VD $=56(49.55 \%)$ C-section $=57(50.44 \%)$ \\
Number of parturitions & $1^{\text {st }}=108(95.57 \%)$ \\
& $2^{\text {nd }}=3(2.65 \%)$ \\
& $3^{\text {rd }}=2(1.77 \%)$ \\
Hospital & HR $1^{\circ}$ October $=35(30.97 \%)$ \\
& HRAEl $=40(35.4 \%)$ \\
Clinical group & HMuj= $38(33.63 \%)$ \\
(Data from documented antigenic & Women without documented infectious events $=63(55.75 \%)$ \\
stimulation during pregnancy) & Women with documented infectious events $=50(44.25 \%)$ \\
& Women who received TT vaccination $=27(23.9 \%)$ \\
& Women who received PPS vaccination $=98(86.72 \%)$ \\
\hline
\end{tabular}

TT, tetanus toxin; PPS, pneumococcal polysaccharide; VD, vaginal delivery; C-section, cesarean section.

chronic maternal disease, previous abortions, preterm pregnancy, multiparous parturition, women with hormonal treatment, or acute disease within 3 weeks before delivery.

\section{Sample processing}

Colostrum samples were collected from clinically healthy women between 1 and $12 \mathrm{~h}$ post-delivery and frozen at $-20^{\circ} \mathrm{C}$ until use. After defrosting at room temperature, all samples were centrifuged at $2000 \mathrm{~g}$ for $10 \mathrm{~min}$ at $4^{\circ} \mathrm{C}$ (Allegra ${ }^{\mathrm{TM}} \mathrm{X}-22 \mathrm{R}$ Benchtop Centrifuges Beckman Coulter Life Sciences, Indianapolis, IN, USA) to prepare the corresponding dilutions (see below).

\section{ELISA for quantification of specific Igs}

A modification of the quantitative ELISA previously reported ${ }^{10}$ was used to quantify $\lg A 1$ and $\lg A 2$ concentrations against TT, PPS, or LPS in colostrum. Anti-TT antibodies quantification: ninety-six-well plates (Immuno Plate MaxiSorp, Thermo Scientific, NY, USA, Cat. Num. 1132249) were coated with 50 $\mu \mathrm{L}$ of $0.5 \mu \mathrm{g} / \mathrm{mL}$ of antigen (TT from C. tetani, SigmaAldrich $^{\oplus}$, Darmstadt, Germany, Cat. Num. T319425UG) in borate buffer. Plates were incubated overnight at $4^{\circ} \mathrm{C}$. Plates were washed with $100 \mu \mathrm{L}$ of $0.05 \%$ polyoxyethylene sorbitan monolaurate (Tween 20, Sigma-Aldrich Company ${ }^{\circledR}$, USA, Cat. Num. $9005-$ 64-5) in phosphate-buffered saline (PBS) (PBS-T) for 8 times. The non-specific binding free sites were blocked with $100 \mu \mathrm{L}$ of $5 \%$ skim milk formula (Svelty ${ }^{\circledR}$ diluted in PBS) by incubation during $60 \mathrm{~min}$ at $37^{\circ} \mathrm{C}$. Anti-PPS antibodies quantification: plates were coated with $50 \mu \mathrm{L}$ of $5 \mu \mathrm{g} / \mathrm{mL}$ of total antigen (capsular polysaccharides from 23 pneumococcal serotypes, Pulmovax ${ }^{\circledR}$, Schering-Plough ${ }^{\oplus}$, West Point, PA, USA, Lot. Num. R033367) in PBS; plates were incubated during $5 \mathrm{~h}$ at $37^{\circ} \mathrm{C}$ and then overnight at $4^{\circ} \mathrm{C}$. Plates were washed with $100 \mu \mathrm{L}$ of PBS-T for 5 times. The non-specific binding free sites were blocked with $5 \%$ of bovine serum albumin (BSA, Sigma-Aldrich Company ${ }^{\circledR}$, USA, Cat. Num. 9048-46-8) with serum fetal bovine (SFB, Biowest ${ }^{\oplus}$, Riverside, MO, USA, Cat. Num. US1520) diluted in PBS-T (200 $\mu$ Ler well) by incubation during $60 \mathrm{~min}$ at $37^{\circ} \mathrm{C}$. Anti-LPS antibodies quantification: plates were coated with $50 \mu \mathrm{L}$ of $5 \mu \mathrm{g} / \mathrm{mL}$ of antigen (LPS from Escherichia coli, SigmaAldrich ${ }^{\oplus}$, Darmstadt, Germany, Cat. Num. L2143) in PBS. Plates were incubated overnight at $4^{\circ} \mathrm{C}$ and washed with $100 \mu \mathrm{L}$ of PBS-T for 5 times. The nonspecific binding free sites were blocked with $5 \%$ BSA diluted in PBS-T ( $200 \mu \mathrm{L}$ per well) by incubation for 30 min at $37^{\circ} \mathrm{C}$. 
From this point, all plates were processed under the same conditions: centrifuged samples were processed by 10 -fold serial dilutions starting at ratios of $1: 10$, $1: 100,1: 1000$, and 1:10,000. For standard curves, 50 $\mu \mathrm{L}$ of standard serum dilutions from healthy individuals vaccinated against TT, PPC, or reference human serum to LPS were added and incubated for $2 \mathrm{~h}$ at $37^{\circ} \mathrm{C}$. For the detection of specific IgA subclasses, 50 $\mu \mathrm{L}$ per well of biotinylated antibodies anti-lgA1 (monoclonal mouse antibody, Abcam ${ }^{\oplus}$, Cat. Num. ab99796; 1:2500) or anti-lgA2 (monoclonal mouse antibody, Abcam ${ }^{\oplus}$, Cat. Num. ab128731; 1:2000) were added and incubated under the same experimental conditions. Then, $50 \mu \mathrm{L}$ of streptavidin-horseradish peroxidase (HRP) complex (Streptavidin HRP, Abcam $^{\circledast}$, Cat. Num. ab7403) dilution were added and incubated for $1 \mathrm{~h}$ at $37^{\circ} \mathrm{C}$. The presence of labeled antibodies was revealed using $50 \mu \mathrm{L}$ per well of chromogenic substrate 3'3"-5-5-tetramethylbenzidine (TMB ELISA Substrate, Abcam ${ }^{\circledR}$, Cat. Num. 171523) and the reaction was stopped by adding $50 \mu \mathrm{L}$ of 0.2 $M$ sulfuric acid per well. The absorbance was measured in a Microplate ELISA plate reader spectrophotometer (Sunrise, Tecan $^{\circledR}$ TX, USA) at $450 \mathrm{~nm}$. IgA1 and IgA2 standard curves were obtained for each plate to determine each sample concentration by absorbance interpolation and dilution factor. Results were finally expressed in nanograms of each IgA subclass per milliliter of colostrum $(\mathrm{ng} / \mathrm{mL})$.

\section{Western blot}

The ability of colostrum to recognize protein antigens from $E$. coli was evaluated by Western blot. For these assays, 10 representative colostrum samples were evaluated. E. coli O157:H7 from the microbial collection of the National School of Biological Sciences-IPN (Mexico) was grown overnight in LB medium without antibiotics. Bacteria were lysed for $30 \mathrm{~min}$ at $4^{\circ} \mathrm{C}$ with RIPA buffer (20 mM Tris-HCl pH 7.5, $150 \mathrm{mM} \mathrm{NaCl}$, $1 \mathrm{mM}$ Na2 EDTA, 1 mM EGTA, $1 \%$ NP-40, $1 \%$ sodium deoxycholate, $2.5 \mathrm{mM}$ sodium pyrophosphate, $1 \mathrm{mM}$ b-glycerophosphate, $1 \mathrm{mM} \mathrm{Na}_{3} \mathrm{VO}_{4}$, and $1 \mu \mathrm{g} / \mathrm{ml}$ leupeptin). The lysate was then centrifuged at 12,000 $\times g$ for 15 min at $4^{\circ} \mathrm{C}$. The supernatant was separated and quantified by the Lowry method, 6 X Laemmli buffer was added to the proteins, and samples were heated at $95^{\circ} \mathrm{C}$ for $5 \mathrm{~min}$. Two-hundred micrograms of proteins were loaded and separated in $10 \%$ polyacrylamide preparative gel. The proteins were transferred to a nitrocellulose membrane $(0.45 \mu \mathrm{m}$; BIO-RAD Cat. Num. 1620115) by a humid chamber (BIO-RAD Mini Trans-Blot ${ }^{\circledR}$ Cell Cat. Num. 1703930) at 100 volts for $1.5 \mathrm{~h}^{14}$. Non-specific binding free sites in the membrane were blocked with $5 \%$ skim milk formula (Svelty ${ }^{\circledR}$ diluted in PBS) by incubation during $60 \mathrm{~min}$ at $37^{\circ} \mathrm{C}$. Colostrum dilutions (1:1000) in $0.05 \%$ PBS-T were used as the primary antibody, and the membrane was incubated overnight at $4^{\circ} \mathrm{C}$. Biotinylated antibodies such as anti-total IgA (rabbit polyclonal antibody, Abcam ${ }^{\oplus}$, Cat. Num. ab97218; 1:5000), anti-lgA1 (Abcam ${ }^{\circledR}$, Cat. Num. ab99796; 1:2500), or anti-lgA2 (Abcam ${ }^{\oplus}$, Cat. Num. ab128731; $1: 2000$ ) in $0.05 \%$ PBS-T were added and incubated 1 $\mathrm{h}$ at room temperature. Streptavidin-HRP was used at 1:8000 dilution and incubated $1 \mathrm{~h}$ at room temperature. Clarity Western ECL (luminol and peroxide) was used as a substrate. Membranes were documented using ChemiDoc ${ }^{\mathrm{TM}}$ MP Imaging System with Image Lab $^{\text {TM }}$ Software.

\section{Statistical analysis}

The IgA concentration distribution was analyzed by the Kolmogorov-Smirnov test. A comparison of Ig concentrations between samples was performed by non-parametric Mann-Whitney U-test or KruskalWallis test. All statistical tests and graphics were developed using statistical program GraphPad Prism (GraphPad ${ }^{\circledR}$ Software version 5, La Jolla, CA, USA).

\section{RESULTS}

\section{Population data}

The mean age of mothers was $27.4 \pm 5.2$ years; $50 \%$ had a cesarean delivery ( $C$-section) and the other half a VD. For $95 \%$ of women, this was their first child. Information on previous infectious episodes and vaccine protocols during pregnancy is shown in Table 1.

\section{Levels of IgA subclasses in colostrum against studied antigens}

The levels of IgA subclasses against TT, PPS, and LPS were quantified in colostrum. None of the colostrum Ig levels had a normal distribution. As in the previous reports ${ }^{7}$, we found that colostrum had higher amounts of IgA1 against TT and, in contrast, IgA2 was more abundant against PPS and LPS (Fig. 1). 
Figure 1. Immunoglobulin (Ig)A subclasses in the function of the type of antigen. The bar graph shows the concentrations of IgA1 (blue) and IgA2 (red) against tetanus toxin, Escherichia coli lipopolysaccharide, and Pulmovax $23^{\circledR}$ (pneumococcal polysaccharides $(n=113)$. All values are represented as mean \pm standard deviation. All data are expressed in nanograms of Ig/milliliter of colostrum $(\mathrm{ng} / \mathrm{mL})$. Statistical analysis was performed using the Mann-Whitney U-test. ${ }^{* * *} \mathrm{p}<0.001$.
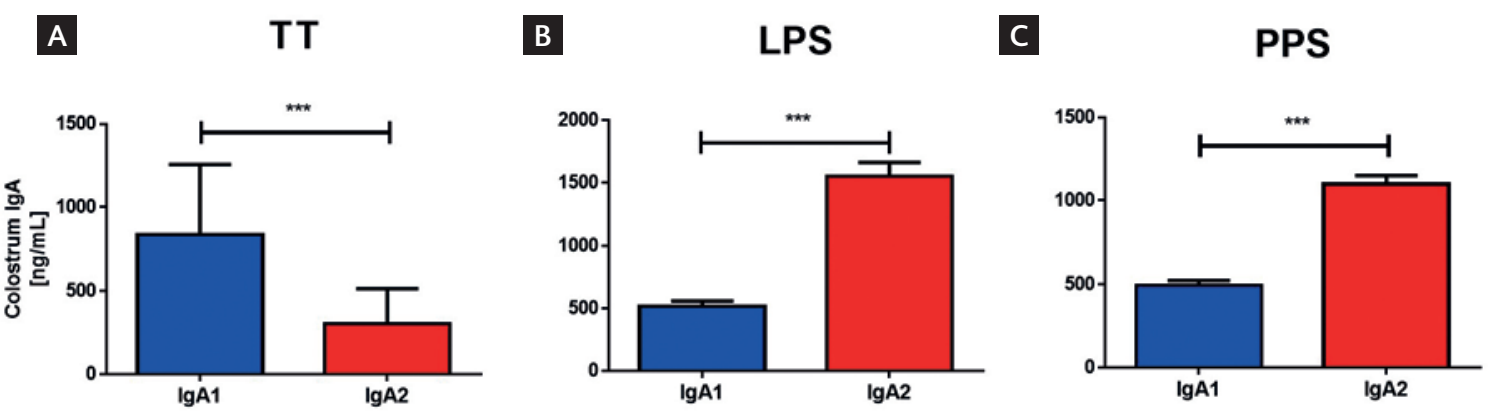

Figure 2. The SDS-PAGE analysis of colostrum immunoglobulin (Ig)A subclasses against Escherichia coli antigens. Colostrum samples were analyzed to determine the recognition profile of E. coli O157:H7 proteins. The membranes were developed with anti-total IgA (A), anti-IgA1 (B), and anti-lgA2 (C). MW markers, for size comparison, are shown at the left. Lanes 1-10 contain 10 selected colostrum samples.

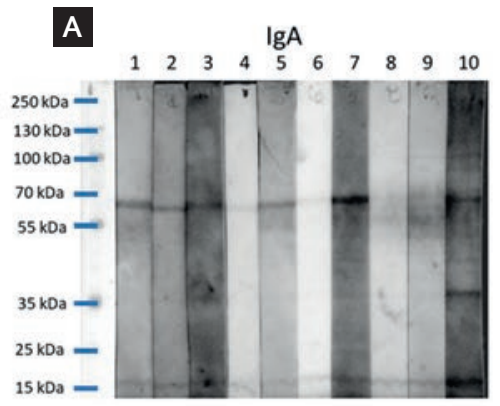

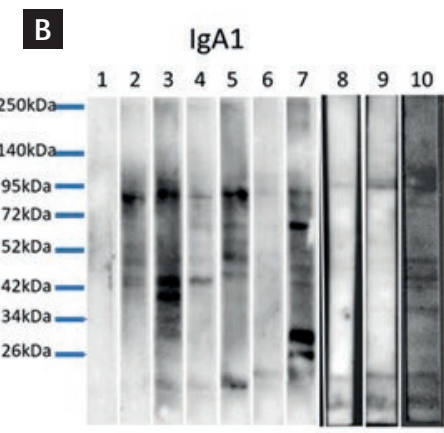

\section{IgA subclasses against $E$. coli antigens by Western blot}

The ability of colostrum to recognize protein antigens from E. coli was evaluated by Western blot. For these assays, 10 representative colostrum samples were evaluated. The pattern of proteins recognized by total IgA was similar among the different colostrum samples, showing variations in the intensity of the bands between the different samples (Fig. 2).

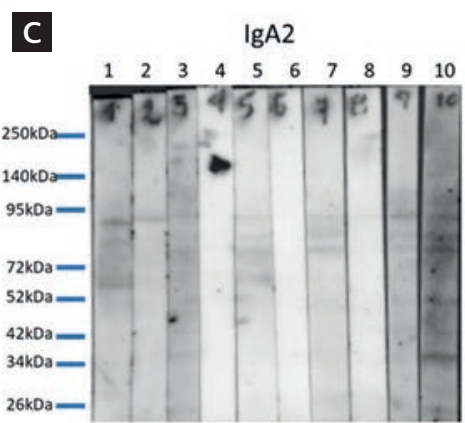

When IgA1 recognition was evaluated, we found that the banding pattern changed considerably; although some samples varied in the number of proteins recognized, and in the intensity of the bands, the pattern was considerably different when compared with total IgA. In sharp contrast, IgA2 recognized fewer antigen proteins with reduced intensity. The pattern and the intensity of the bands recognized by $\lg A 2$ differed from that seen with $\lg A 1$ and total IgA. 
Figure 3. Distribution of immunoglobulin (Ig)A subclasses in the function of the type of antigen, comparing women with or without previous specific antigen stimulation. The amounts of Ig from women with previous infections at the gastrointestinal tract (lipopolysaccharide [LPS]) or vaccination during pregnancy (tetanus toxin [TT] or pneumococcal polysaccharides [PPS]) are compared with each IgA subclass. The bar graphs show IgA1 (blue) and IgA2 (red) between women without documented stimulation during pregnancy (bars with rods $n=63$ ) and women with at least one antigenic stimulation during their pregnancy (bars without rods $=50)$ : TT $(n=27)$ or PPS $(n=98)$ vaccination or LPS $(n=13)$. All values are represented as mean \pm standard deviation. All data are expressed in nanograms of Ig per milliliter of colostrum ( $\mathrm{ng} / \mathrm{mL}$ ). Statistical analysis was performed using the Mann-Whitney U-test. ${ }^{* *} \mathrm{p}<0.001$.

A

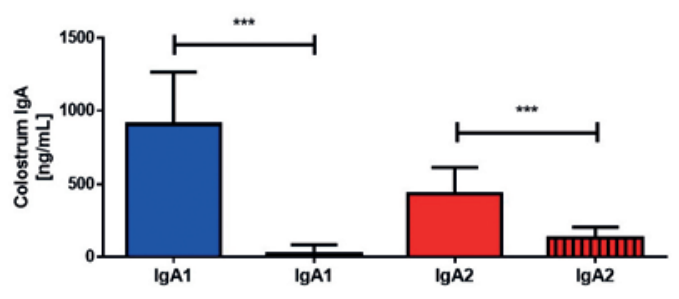

C
B

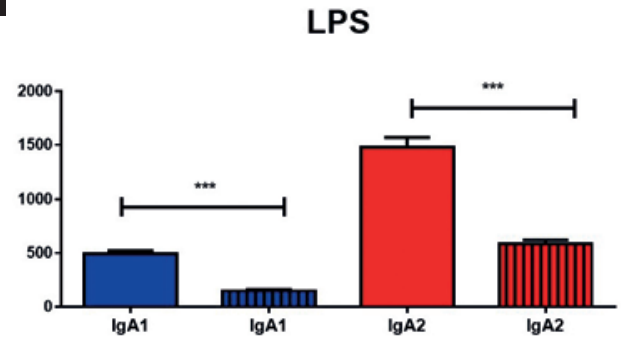

PPS

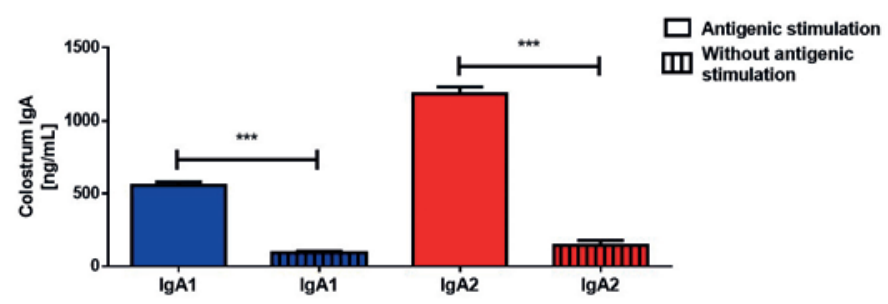

\section{IgA subclasses did not show variations among population descriptors}

Many descriptors played a pivotal role in identifying the characteristics of the population analyzed. The following descriptors were included in the function of the size of the sampled population: the hospital where the mothers were recruited (Supplementary Fig. S1a) and the delivery method (Supplementary Fig. S1b). These comparisons did not show any statistical differences between groups. Hence, we concluded that lg levels were independent of the descriptors used to stratify this population.

\section{The concentrations of colostrum IgA subclasses vary in women with previous antigenic stimulation during pregnancy}

The amount of colostrum IgA subclasses was compared between women without vaccination or previous infection reports during pregnancy. Results showed that both IgA subclasses against TT, PPS, or LPS increased in women vaccinated with specific antigens (TT or PPS) or women that had infections at the gastrointestinal tract during pregnancy, in comparison with women without infections (Fig. 3). These results were compared with total IgA subclasses levels in the colostrum (Supplementary Fig. S2). This effect was analyzed by the Pearson's correlation test to evaluate whether multiple interactions with the same antigen during pregnancy had an effect on IgA subclasses levels in colostrum. Although we did not find a statistical correlation, levels of $\lg A 1$ tended to increase in colostrum from women with more than one TT vaccination dose. In contrast, IgA2 increased in colostrum from women with multiple episodes of gastrointestinal tract infections during pregnancy when LPS was used as an antigen in the test (Supplementary Fig. S3). As it was already mentioned, 98 women vaccinated with Pulmovax ${ }^{\circledR}$ developed a strong response against polysaccharide, with predominant recognition by IgA2 (Fig. 3). 


\section{DISCUSSION}

According to this study, we found that the previous antigenic exposures during pregnancy affected specific $\lg A 1$ and $\lg A 2$ concentrations ${ }^{7}$. However, the mechanisms involved to explain these differences were not studied. As seen from our results, the production of IgA subclasses depends on the chemical nature of the antigen. The humoral responses against most proteins require the interaction of $B$ cells with helper $T$ cells in a thymus-dependent (TD) fashion. $B$-cell activation by TD antigens requires, among many factors, the interaction between CD40 on Bcells and CD4OL on activated Th cells in the germinal centers of the lymph nodes ${ }^{15}$.

On the other hand, a typical abundant polysaccharide (as in the capsule of Gram-positive bacteria or the external membrane of Gram-negative bacteria) is made up of repeating sequences of few simple monosaccharides, which means that there are multiple copies of identical antigenic determinants. When specific naïve B-cells come in contact with such antigens, these antigenic determinants bind the surface lgM and IgD receptors. This binding leads to the clustering of surface lgs, which generates a signal strong enough to activate the naïve B-cells. Another way to activate $B$-cells is by the clustering of repetitive epitopes and the stimulation by TLR signals, although in the case of human B-cells, they have low levels of TLR4 on their surface ${ }^{16}$. $T$-independent activated B-cells produce and release IgM and "natural" IgA ${ }^{17}$. The previous reports have suggested that the cells producing IgA2 are mainly found in the gastrointestinal tract, and these cells are predominantly activated through T-independent mechanisms ${ }^{18,19}$.

A remarkable result, derived from the recognition of E. coli proteins by IgA subclasses, showed (as expected) variations between different colostrum samples, but interestingly, also exhibited differences between $\lg A 1, \lg A 2$, and total $\lg A$ recognition. To get a more interesting contrast, when IgA1 mainly recognized the proteins from the lysates, IgA2 displayed a more pronounced reactivity against LPS, contrasting the nature of TD versus $\mathrm{TI}$ antigen stimulation in the production of IgA subclasses.

During the final trimester of pregnancy, these $\lg A 1$ or IgA2 producing cells migrate to mammary glands under hormonal regulation ${ }^{20}$ and the expression of different adhesion molecules ${ }^{21,22}$. These cells can then reside in the mammary gland until lactation ${ }^{23,24}$, where they produce high levels of IgA subclasses into the mammary acini.

This study has some limitations. It is based on previous antigen stimulation that women recalled and reported during pregnancy. For the correlations, vaccinations (reported in the clinical files) are more reliable parameters for the analysis. Thus, a more systematic clinical study is necessary to demonstrate a correlation between clinical variables and their role in the maternal transference of specific IgA subclasses in mothers and babies. Despite the limitations described above, the results derived from this research provide data and additional information that contribute to solve the controversial findings about the effect of infections during pregnancy on IgA production in the colostrum. This study provides pieces of evidence to understand the effect of infections on the production of IgA subclasses during pregnancy. Moreover, the results offer experimental confirmation in which interactions with specific antigens during pregnancy modify the levels of specific $\lg A 1$ and $\lg A 2$ in colostrum.

\section{ACKNOWLEDGEMENTS}

The authors thank the women who participated in this study. The authors also thank Kaleb Geovanni Mondragón-Ramírez, Héctor Armando Guzmán-Aquino, María Fernanda de la Borbolla, Zeltzin Arisvet Mendoza-Juárez, and Maria Inés Valdivia-Hernández who participated at different stages of this work.

Dr. Santos-Argumedo reports receiving the following grants during the development of the study: Consejo Nacional de Ciencia y Tecnología (CONACyT [PDCPN 2015/900]), Fundación Miguel Alemán, Mexico (2018). Scholarship 727506 was granted to Carlos E. Miguel-Rodríguez and Scholarship 592631 to Erick S. Sánchez-Salguero, both from CONACyT, Mexico. This study protocol is an extension of a previous protocol that was approved by the Research and Ethics Reviewing Board of Protocols from Hospital Regional $1^{\circ}$ de Octubre, ISSSTE, Mexico City, Mexico (with number 090201/14.1/086/2017), Hospital Regional de Alta 
Especialidad de Ixtapaluca (HRAEI), Ixtapaluca, Mexico (FESZ/DEP/Cl/163/16), and Hospital de la Mujer (HMuj), SSA, Mexico City (HM-INV/2016-015), Mexico.

\section{SUPPLEMENTARY DATA}

Supplementary data are available at Revista de Investigación Clínica online (www.clinicalandtranslationalinvestigation.com). These data are provided by the corresponding author and published online for the benefit of the reader. The contents of supplementary data are the sole responsibility of the authors.

\section{REFERENCES}

1. De Schutter S, Maertens K, Baerts L, De Meester I, Van Damme $P$, Leuridan E. Quantification of vaccine-induced antipertussis toxin secretory IgA antibodies in breast milk: comparison of different vaccination strategies in women. Pediatr Infect Dis J. 2015;34:e149-52

2. Obaro SK, Deubzer HE, Newman VO, Adegbola RA, Greenwood BM, Henderson DC. Serotype-specific pneumococcal antibodies in breast milk of Gambian women immunized with a pneumococcal polysaccharide vaccine during pregnancy. Pediatr Infect Dis ]. 2004:23:1023-9.

3. Hoffman JA, Mason EO, Schutze GE, Tan TQ, Barson WJ, Givner $\mathrm{LB}$, et al. Streptococcus pneumoniae infections in the neonate. Pediatrics. 2003;112:1095-102.

4. Maertens K, De Schutter S, Braeckman T, Baerts L, Van Damme $P$, De Meester I, et al. Breastfeeding after maternal immunisation during pregnancy: providing immunological protection to the newborn: a review. Vaccine. 2014;32:1786-92.

5. Brandtzaeg P. Secretory IgA: designed for anti-microbial defense. Front Immunol. 2013;4:222.

6. Fagarasan S, Kawamoto S, Kanagawa O, Suzuki K. Adaptive immune regulation in the gut: $\mathrm{T}$ cell-dependent and $\mathrm{T}$ cell-independent IgA synthesis. Annu Rev Immunol. 2010;28:243-73.

7. Ladjeva I, Peterman JH, Mestecky J. IgA subtypes of human colostral antibodies specific for microbial and food antigens. Clin Exp Immunol. 1989;78:85-90.

8. Munoz FM, Jamieson DJ. Maternal immunization. Obstet Gynecol. 2019;133:739-53.
9. Marchant A, Sadarangani M, Garand M, Dauby N, Verhasselt V, Pereira L, et al. Maternal immunisation: collaborating with mother nature. Lancet Infect Dis. 2017;17:e197-208.

10. Sánchez-Salguero E, Mondragón-Ramírez GK, Alcántara-Montie JC, Cérbulo-Vázquez A, Villegas-Domínguez $X$, ContrerasVargas $V M$, et al. Infectious episodes during pregnancy, at particular mucosal sites, increase specific IgA1 or IgA2 subtype levels in human colostrum. Matern Health Neonatol Perinatol. 2019;5:9.

11. Di Lorenzo F, De Castro C, Silipo A, Molinaro A. Lipopolysaccharide structures of gram-negative populations in the gut microbiota and effects on host interactions. FEMS Microbiol Rev. 2019;43:257-72

12. Demers-Mathieu V, Underwood MA, Beverly RL, Nielsen SD, Dallas DC. Comparison of human milk immunoglobulin survival during gastric digestion between preterm and term infants. Nutrients. 2018:10:E631.

13. World Medical Association. World medical association declaration of Helsinki: ethical principles for medical research involving human subjects. JAMA. 2013;310:2191-4.

14. Da Silva WM, Bei J, Amigo N, Valacco MP, Amadio A, Zhang Q, et al. Quantification of enterohemorrhagic Escherichia coli O157: H7 protein abundance by high-throughput proteome. PLoS One. 2018;13:e0208520.

15. Bunker JJ, Bendelac A. IgA responses to microbiota. Immunity. 2018;49:211-24

16. Ganley-Leal LM, Liang Y, Jagannathan-Bogdan M, Farraye FA, Nikolajczyk BS. Differential regulation of TLR4 expression in human B cells and monocytes. Mol Immunol. 2010;48:82-8

17. Mathias A, Corthésy B. Recognition of gram-positive intestinal bacteria by hybridoma-and colostrum-derived secretory immunoglobulin A is mediated by carbohydrates. J Biol Chem. 2011; 286:17239-47

18. He B, Xu W, Santini PA, Polydorides AD, Chiu A, Estrella J, et al. Intestinal Bacteria trigger $T$ cell-independent immunoglobulin $A(2)$ class switching by inducing epithelial-cell secretion of the cytokine APRIL. Immunity. 2007;26:812-26.

19. Woof JM, Russell MW. Structure and function relationships in IgA. Mucosal Immunol. 2011;4:590-7.

20. Brisken C, O'Malley B. Hormone action in the mammary gland. Cold Spring Harb Perspect Biol. 2010;2:a003178.

21. Seong $Y$, Lazarus $N H$, Sutherland $L$, Habtezion $A$, Abramson $T$, He XS, et al. Trafficking receptor signatures define blood plasmablasts responding to tissue-specific immune challenge. $\mathrm{JCl}$ Insight. 2017;2:e90233.

22. Pakkanen SH, Kantele JM, Moldoveanu Z, Hedges S, Häkkinen M, Mestecky J, et al. Expression of homing receptors on IgA1 and IgA2 plasmablasts in blood reflects differential distribution of IgA 1 and IgA2 in various body fluids. Clin Vaccine Immunol. 2010;17:393-401.

23. Bourges D, Meurens F, Berri M, Chevaleyre C, Zanello G, Levast B, et al. New insights into the dual recruitment of lgA+B cells in the developing mammary gland. Mol Immunol. 2008;45:3354-62.

24. Low EN, Zagieboylo L, Martino B, Wilson E. IgA ASC accumulation to the lactating mammary gland is dependent on VCAM-1 and alpha4 integrins. Mol Immunol. 2010;47:1608-12. 